Supporting Information Available: Tables of crystallographic data, and bond lengths and angles

\title{
Dehydrogenation of a Coordinated Alkylphosphine as Method to Prepare Cyclopentadienyl- $\alpha$, alkenylphosphine-osmium Complexes
}

Miguel Baya, María L. Buil, Miguel A. Esteruelas, and Enrique Oñate. 
Table 1. Crystal data and structure refinement for 5 .

\begin{tabular}{|c|c|}
\hline Identification code & 5 \\
\hline Empirical formula & C28 H36 F6 Os P2 \\
\hline Formula weight & 738.71 \\
\hline Temperature & $100(2) \mathrm{K}$ \\
\hline Wavelength & $0.71073 \mathrm{~A}$ \\
\hline Crystal system, space group & Monoclinic, $\quad$ P $121 / n 1$ \\
\hline Unit cell dimensions & $\begin{array}{l}\mathrm{a}=11.2563(9) \text { A } \quad \text { alpha }=90 \text { deg. } \\
\mathrm{b}=20.2337(16) \text { A beta }=92.3440(10) \text { deg. } \\
\mathrm{c}=12.3703(10) \text { A gamma }=90 \text { deg. }\end{array}$ \\
\hline Volume & $2815.1(4) A^{\wedge} 3$ \\
\hline Z, Calculated density & 4, $1.743 \mathrm{Mg} / \mathrm{m}^{\wedge} 3$ \\
\hline Absorption coefficient & $4.700 \mathrm{~mm}^{\wedge}-1$ \\
\hline$F(000)$ & 1456 \\
\hline Crystal size & $0.20 \times 0.12 \times 0.12 \mathrm{~mm}$ \\
\hline Theta range for data collection & 1.93 to $28.52 \mathrm{deg}$. \\
\hline Limiting indices & $-14<=\mathrm{h}<=15, \quad-26<=\mathrm{k}<=26, \quad-15<=1<=16$ \\
\hline Reflections collected / unique & $33606 / 6741[R($ int $)=0.0397]$ \\
\hline Completeness to theta $=28.52$ & $94.5 \%$ \\
\hline Absorption correction & Semi-empirical from equivalents \\
\hline Max. and min. transmission & 0.608 and 0.445 \\
\hline Refinement method & Full-matrix least-squares on $\mathrm{F}^{\wedge} 2$ \\
\hline Data / restraints / parameters & $6741 / 0 / 351$ \\
\hline Goodness-of-fit on $\mathrm{F}^{\wedge} 2$ & 0.984 \\
\hline Final R indices [I>2sigma(I)] & $\mathrm{R} 1=0.0319, \mathrm{wR} 2=0.0694$ \\
\hline $\mathrm{R}$ indices (all data) & $\mathrm{R} 1=0.0418, \mathrm{wR} 2=0.0718$ \\
\hline Largest diff. peak and hole & 4.923 and -0.976 e. $A^{\wedge}-3$ \\
\hline
\end{tabular}


Table 2. Atomic coordinates $\left(\mathrm{x} 10^{\wedge} 4\right)$ and equivalent isotropic displacement parameters $\left(A^{\wedge} 2 \times 10^{\wedge} 3\right)$ for 5 .

$\mathrm{U}(\mathrm{eq})$ is defined as one third of the trace of the orthogonalized Uij tensor.

\begin{tabular}{|c|c|c|c|c|}
\hline & $x$ & $\mathrm{Y}$ & $z$ & $\mathrm{U}(\mathrm{eq})$ \\
\hline Os & $598(1)$ & $1985(1)$ & $9677(1)$ & $16(1)$ \\
\hline $\mathrm{P}(1)$ & $916(1)$ & $3068(1)$ & $10205(1)$ & $17(1)$ \\
\hline$P(2)$ & $5852(1)$ & 2971 (1) & $9505(1)$ & $24(1)$ \\
\hline$F(1)$ & $5508(3)$ & $3723(1)$ & $9689(2)$ & $43(1)$ \\
\hline$F(2)$ & $6697(3)$ & $3170(2)$ & $8555(2)$ & $54(1)$ \\
\hline$F(3)$ & $6173(3)$ & $2213(1)$ & $9307(3)$ & $47(1)$ \\
\hline$F(4)$ & $5012(2)$ & $2760(2)$ & $10449(2)$ & $36(1)$ \\
\hline$F(5)$ & $4750(2)$ & $2902(1)$ & $8640(2)$ & $36(1)$ \\
\hline$F(6)$ & $6940(2)$ & $3033(1)$ & $10369(2)$ & $41(1)$ \\
\hline$C(1)$ & 1901 (4) & $1593(2)$ & $8578(3)$ & $19(1)$ \\
\hline$C(2)$ & $1725(3)$ & $909(2)$ & 8171 ( 3 ) & $20(1)$ \\
\hline$C(3)$ & $2094(4)$ & $329(2)$ & $8698(3)$ & $20(1)$ \\
\hline$C(4)$ & $1884(4)$ & $-280(2)$ & $8232(3)$ & $22(1)$ \\
\hline$C(5)$ & $1310(4)$ & $-341(2)$ & $7240(3)$ & $26(1)$ \\
\hline$C(6)$ & $952(4)$ & $228(2)$ & $6694(3)$ & $29(1)$ \\
\hline$C(7)$ & $1160(4)$ & $841(2)$ & 7151 ( 3 ) & $25(1)$ \\
\hline$C(8)$ & $2499(4)$ & $1818(2)$ & $9560(3)$ & $20(1)$ \\
\hline$C(9)$ & $3245(4)$ & $1403(2)$ & $10342(3)$ & $21(1)$ \\
\hline$C(10)$ & $4287(4)$ & $1128(2)$ & $9977(4)$ & $26(1)$ \\
\hline$C(11)$ & $5017(4)$ & $753(2)$ & $10651(4)$ & $31(1)$ \\
\hline$C(12)$ & $4730(4)$ & $644(2)$ & $11711(4)$ & $33(1)$ \\
\hline$C(13)$ & $3716(4)$ & $926(2)$ & 12091 ( 4) & $32(1)$ \\
\hline$C(14)$ & $2985(4)$ & $1309(2)$ & $11412(3)$ & $25(1)$ \\
\hline$C(15)$ & $825(4)$ & $2953(2)$ & $8790(3)$ & $20(1)$ \\
\hline$C(16)$ & $1791(4)$ & $3130(2)$ & 8029 (3) & $22(1)$ \\
\hline$C(17)$ & $-221(4)$ & $2635(2)$ & $8417(4)$ & $22(1)$ \\
\hline$C(18)$ & $2347(4)$ & $3428(2)$ & $10645(3)$ & $19(1)$ \\
\hline C (19) & $2663(4)$ & $3257(2)$ & $11820(4)$ & $32(1)$ \\
\hline$C(20)$ & $2455(4)$ & $4167(2)$ & $10444(4)$ & $33(1)$ \\
\hline$C(21)$ & $-370(4)$ & $3507(2)$ & 10701 (3) & $21(1)$ \\
\hline$C(22)$ & $-428(4)$ & $3477(2)$ & $11936(4)$ & $35(1)$ \\
\hline$C(23)$ & $-511(4)$ & $4221(2)$ & $10283(4)$ & $30(1)$ \\
\hline$C(24)$ & $-12(4)$ & $1538(2)$ & 11178 (3) & $22(1)$ \\
\hline$C(25)$ & $-1025(4)$ & $1870(2)$ & $10672(4)$ & $23(1)$ \\
\hline$C(26)$ & $-1297(4)$ & $1546(2)$ & $9681(3)$ & $24(1)$ \\
\hline$C(27)$ & $-465(4)$ & $1038(2)$ & $9533(3)$ & $24(1)$ \\
\hline$C(28)$ & $329(4)$ & $1026(2)$ & $10459(3)$ & $22(1)$ \\
\hline
\end{tabular}


Table 3. Bond lengths [A] and angles [deg] for 5.

\begin{tabular}{|c|c|c|c|}
\hline $\mathrm{Os}-\mathrm{C}(8)$ & $2.177(4)$ & $\mathrm{C}(19)-\mathrm{H}(19 \mathrm{C})$ & 0.9800 \\
\hline $\mathrm{Os}-\mathrm{C}(1)$ & $2.189(4)$ & $\mathrm{C}(20)-\mathrm{H}(20 \mathrm{~A})$ & 0.9800 \\
\hline $\mathrm{Os}-\mathrm{C}(28)$ & $2.195(4)$ & $\mathrm{C}(20)-\mathrm{H}(20 \mathrm{~B})$ & 0.9800 \\
\hline $\mathrm{Os}-\mathrm{C}(24)$ & $2.201(4)$ & $\mathrm{C}(20)-\mathrm{H}(20 \mathrm{C})$ & 0.9800 \\
\hline $\mathrm{Os}-\mathrm{C}(17)$ & $2.211(4)$ & $C(21)-C(22)$ & $1.534(6)$ \\
\hline $\mathrm{Os}-\mathrm{C}(25)$ & $2.256(4)$ & $C(21)-C(23)$ & $1.540(6)$ \\
\hline $\mathrm{Os}-\mathrm{C}(27)$ & $2.261(4)$ & $\mathrm{C}(21)-\mathrm{H}(21)$ & 1.0000 \\
\hline $\mathrm{Os}-\mathrm{C}(15)$ & $2.264(4)$ & $\mathrm{C}(22)-\mathrm{H}(22 \mathrm{~A})$ & 0.9800 \\
\hline $\mathrm{Os}-\mathrm{P}(1)$ & $2.3093(10)$ & $\mathrm{C}(22)-\mathrm{H}(22 \mathrm{~B})$ & 0.9800 \\
\hline $\mathrm{Os}-\mathrm{C}(26)$ & $2.310(4)$ & $\mathrm{C}(22)-\mathrm{H}(22 \mathrm{C})$ & 0.9800 \\
\hline$P(1)-C(15)$ & $1.765(4)$ & $\mathrm{C}(23)-\mathrm{H}(23 \mathrm{~A})$ & 0.9800 \\
\hline$P(1)-C(21)$ & $1.826(4)$ & $\mathrm{C}(23)-\mathrm{H}(23 \mathrm{~B})$ & 0.9800 \\
\hline$P(1)-C(18)$ & $1.830(4)$ & $\mathrm{C}(23)-\mathrm{H}(23 \mathrm{C})$ & 0.9800 \\
\hline$P(2)-F(1)$ & $1.588(3)$ & $C(24)-C(28)$ & $1.429(6)$ \\
\hline$P(2)-F(4)$ & $1.591(3)$ & $C(24)-C(25)$ & $1.444(6)$ \\
\hline$P(2)-F(2)$ & $1.593(3)$ & $\mathrm{C}(24)-\mathrm{H}(24)$ & 0.9500 \\
\hline$P(2)-F(3)$ & $1.598(3)$ & $C(25)-C(26)$ & $1.412(6)$ \\
\hline$P(2)-F(6)$ & $1.598(3)$ & $\mathrm{C}(25)-\mathrm{H}(25)$ & 0.9500 \\
\hline$P(2)-F(5)$ & $1.610(3)$ & $C(26)-C(27)$ & $1.407(6)$ \\
\hline$C(1)-C(8)$ & $1.439(6)$ & $\mathrm{C}(26)-\mathrm{H}(26)$ & 0.9500 \\
\hline$C(1)-C(2)$ & $1.483(6)$ & $C(27)-C(28)$ & $1.424(6)$ \\
\hline $\mathrm{C}(1)-\mathrm{H}(1)$ & $0.92(4)$ & $\mathrm{C}(27)-\mathrm{H}(27)$ & 0.9500 \\
\hline$C(2)-C(7)$ & $1.396(6)$ & $\mathrm{C}(28)-\mathrm{H}(28)$ & 0.9500 \\
\hline$C(2)-C(3)$ & $1.397(6)$ & & \\
\hline$C(3)-C(4)$ & $1.376(6)$ & $\mathrm{C}(8)-\mathrm{Os}-\mathrm{C}(1)$ & $38.48(15)$ \\
\hline $\mathrm{C}(3)-\mathrm{H}(3)$ & 0.9500 & $C(8)-O s-C(28)$ & $92.63(15)$ \\
\hline$C(4)-C(5)$ & $1.368(6)$ & $C(1)-O s-C(28)$ & $93.51(15)$ \\
\hline $\mathrm{C}(4)-\mathrm{H}(4)$ & 0.9500 & $C(8)-O s-C(24)$ & $109.50(16)$ \\
\hline$C(5)-C(6)$ & $1.385(6)$ & $\mathrm{C}(1)-\mathrm{Os}-\mathrm{C}(24)$ & $127.97(16)$ \\
\hline $\mathrm{C}(5)-\mathrm{H}(5)$ & 0.9500 & $C(28)-O s-C(24)$ & $37.93(15)$ \\
\hline$C(6)-C(7)$ & $1.379(6)$ & $\mathrm{C}(8)-\mathrm{Os}-\mathrm{C}(17)$ & $115.34(16)$ \\
\hline $\mathrm{C}(6)-\mathrm{H}(6)$ & 0.9500 & $\mathrm{C}(1)-\mathrm{Os}-\mathrm{C}(17)$ & $92.78(16)$ \\
\hline $\mathrm{C}(7)-\mathrm{H}(7)$ & 0.9500 & $C(28)-O s-C(17)$ & $140.83(16)$ \\
\hline$C(8)-C(9)$ & $1.510(6)$ & $C(24)-O s-C(17)$ & $134.72(16)$ \\
\hline $\mathrm{C}(8)-\mathrm{H}(8)$ & $0.88(4)$ & $C(8)-O s-C(25)$ & $147.17(16)$ \\
\hline$C(9)-C(14)$ & $1.380(6)$ & $C(1)-O s-C(25)$ & $151.55(15)$ \\
\hline$C(9)-C(10)$ & $1.390(6)$ & $C(28)-O s-C(25)$ & $62.54(15)$ \\
\hline$C(10)-C(11)$ & $1.375(6)$ & $C(24)-O s-C(25)$ & $37.78(15)$ \\
\hline $\mathrm{C}(10)-\mathrm{H}(10)$ & 0.9500 & $C(17)-O s-C(25)$ & $97.03(16)$ \\
\hline$C(11)-C(12)$ & $1.381(6)$ & $C(8)-O s-C(27)$ & $112.43(15)$ \\
\hline $\mathrm{C}(11)-\mathrm{H}(11)$ & 0.9500 & $\mathrm{C}(1)-\mathrm{Os}-\mathrm{C}(27)$ & $90.55(15)$ \\
\hline$C(12)-C(13)$ & $1.377(6)$ & $C(28)-O s-C(27)$ & $37.23(15)$ \\
\hline $\mathrm{C}(12)-\mathrm{H}(12)$ & 0.9500 & $C(24)-O s-C(27)$ & $62.36(15)$ \\
\hline$C(13)-C(14)$ & $1.388(6)$ & $C(17)-O s-C(27)$ & $104.13(16)$ \\
\hline $\mathrm{C}(13)-\mathrm{H}(13)$ & 0.9500 & $C(25)-O s-C(27)$ & $61.18(15)$ \\
\hline $\mathrm{C}(14)-\mathrm{H}(14)$ & 0.9500 & $C(8)-O s-C(15)$ & $88.35(15)$ \\
\hline$C(15)-C(17)$ & $1.403(6)$ & $C(1)-O s-C(15)$ & $85.44(15)$ \\
\hline$C(15)-C(16)$ & $1.509(5)$ & $C(28)-O s-C(15)$ & $176.93(14)$ \\
\hline$C(16)-H(16 A)$ & 0.9800 & $C(24)-O s-C(15)$ & $144.09(15)$ \\
\hline $\mathrm{C}(16)-\mathrm{H}(16 \mathrm{~B})$ & 0.9800 & $C(17)-O s-C(15)$ & $36.51(15)$ \\
\hline $\mathrm{C}(16)-\mathrm{H}(16 \mathrm{C})$ & 0.9800 & $C(25)-O s-C(15)$ & $117.64(14)$ \\
\hline $\mathrm{C}(17)-\mathrm{H}(17 \mathrm{~A})$ & $0.95(4)$ & $C(27)-O s-C(15)$ & $139.82(15)$ \\
\hline $\mathrm{C}(17)-\mathrm{H}(17 \mathrm{~B})$ & $0.87(4)$ & $\mathrm{C}(8)-\mathrm{Os}-\mathrm{P}(1)$ & $91.42(11)$ \\
\hline$C(18)-C(19)$ & $1.521(6)$ & $\mathrm{C}(1)-\mathrm{Os}-\mathrm{P}(1)$ & $114.81(11)$ \\
\hline$C(18)-C(20)$ & $1.522(6)$ & $C(28)-O S-P(1)$ & $137.45(11)$ \\
\hline $\mathrm{C}(18)-\mathrm{H}(18)$ & 1.0000 & $C(24)-O S-P(1)$ & $101.62(11)$ \\
\hline $\mathrm{C}(19)-\mathrm{H}(19 \mathrm{~A})$ & 0.9800 & $\mathrm{C}(17)-\mathrm{Os}-\mathrm{P}(1)$ & $71.97(12)$ \\
\hline $\mathrm{C}(19)-\mathrm{H}(19 \mathrm{~B})$ & 0.9800 & $C(25)-O S-P(1)$ & $93.63(11)$ \\
\hline
\end{tabular}




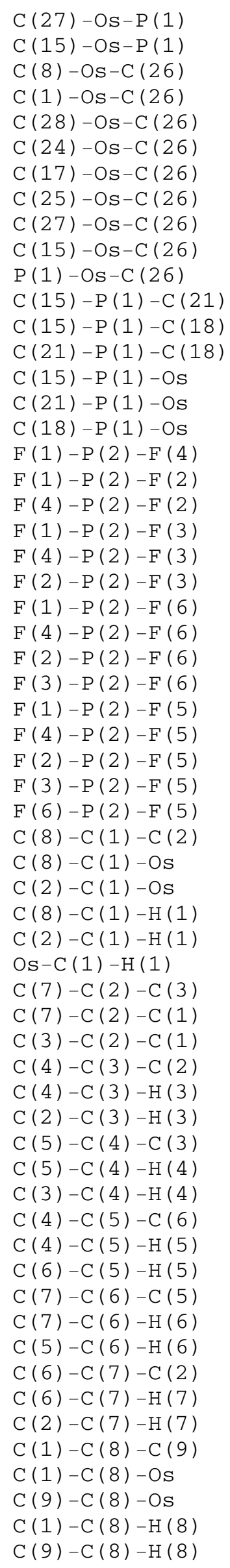

$154.28(11)$

$45.38(10)$

$148.26(16)$

$120.25(15)$

$61.00(15)$

$61.34(15)$

$82.63(16)$

36.01 (15)

$35.83(15)$

$117.12(15)$

$119.75(11)$

$112.52(19)$

111.24 (19)

$114.0(2)$

$65.95(13)$

$116.17(14)$

$125.87(14)$

$89.82(16)$

$91.18(18)$

$179.00(18)$

$178.89(17)$

$89.93(16)$

$89.08(18)$

$90.78(15)$

$89.53(15)$

$90.53(17)$

$90.30(16)$

$89.60(15)$

$90.00(15)$

$89.93(16)$

$89.32(16)$

$179.40(18)$

$129.3(4)$

$70.3(2)$

$117.8(3)$

$117(3)$

$109(3)$

$105(3)$

$117.1(4)$

$116.6(4)$

$126.3(4)$

$120.9(4)$

119.6

119.6

$121.5(4)$

119.2

119.2

$118.7(4)$

120.7

120.7

$120.4(4)$

119.8

119.8

$121.5(4)$

119.3

119.3

$126.4(4)$

$71.2(2)$

$124.4(3)$

$109(3)$

$109(3)$
$\mathrm{Os}-\mathrm{C}(8)-\mathrm{H}(8)$

$\mathrm{C}(14)-\mathrm{C}(9)-\mathrm{C}(10)$

$C(14)-C(9)-C(8)$

$C(10)-C(9)-C(8)$

$C(11)-C(10)-C(9)$

$\mathrm{C}(11)-\mathrm{C}(10)-\mathrm{H}(10)$

$\mathrm{C}(9)-\mathrm{C}(10)-\mathrm{H}(10)$

$\mathrm{C}(10)-\mathrm{C}(11)-\mathrm{C}(12)$

$\mathrm{C}(10)-\mathrm{C}(11)-\mathrm{H}(11)$

$\mathrm{C}(12)-\mathrm{C}(11)-\mathrm{H}(11)$

$\mathrm{C}(13)-\mathrm{C}(12)-\mathrm{C}(11)$

$\mathrm{C}(13)-\mathrm{C}(12)-\mathrm{H}(12)$

$\mathrm{C}(11)-\mathrm{C}(12)-\mathrm{H}(12)$

$C(12)-C(13)-C(14)$

$\mathrm{C}(12)-\mathrm{C}(13)-\mathrm{H}(13)$

$\mathrm{C}(14)-\mathrm{C}(13)-\mathrm{H}(13)$

$C(9)-C(14)-C(13)$

$\mathrm{C}(9)-\mathrm{C}(14)-\mathrm{H}(14)$

$\mathrm{C}(13)-\mathrm{C}(14)-\mathrm{H}(14)$

$\mathrm{C}(17)-\mathrm{C}(15)-\mathrm{C}(16)$

$C(17)-C(15)-P(1)$

$C(16)-C(15)-P(1)$

$\mathrm{C}(17)-\mathrm{C}(15)-\mathrm{OS}$

$\mathrm{C}(16)-\mathrm{C}(15)-\mathrm{OS}$

$\mathrm{P}(1)-\mathrm{C}(15)-\mathrm{OS}$

$\mathrm{C}(15)-\mathrm{C}(16)-\mathrm{H}(16 \mathrm{~A})$

$\mathrm{C}(15)-\mathrm{C}(16)-\mathrm{H}(16 \mathrm{~B})$

$\mathrm{H}(16 \mathrm{~A})-\mathrm{C}(16)-\mathrm{H}(16 \mathrm{~B})$

$\mathrm{C}(15)-\mathrm{C}(16)-\mathrm{H}(16 \mathrm{C})$

$\mathrm{H}(16 \mathrm{~A})-\mathrm{C}(16)-\mathrm{H}(16 \mathrm{C})$

$\mathrm{H}(16 \mathrm{~B})-\mathrm{C}(16)-\mathrm{H}(16 \mathrm{C})$

$\mathrm{C}(15)-\mathrm{C}(17)-\mathrm{OS}$

$\mathrm{C}(15)-\mathrm{C}(17)-\mathrm{H}(17 \mathrm{~A})$

Os $-\mathrm{C}(17)-\mathrm{H}(17 \mathrm{~A})$

$\mathrm{C}(15)-\mathrm{C}(17)-\mathrm{H}(17 \mathrm{~B})$

Os $-\mathrm{C}(17)-\mathrm{H}(17 \mathrm{~B})$

$\mathrm{H}(17 \mathrm{~A})-\mathrm{C}(17)-\mathrm{H}(17 \mathrm{~B})$

$\mathrm{C}(19)-\mathrm{C}(18)-\mathrm{C}(20)$

$\mathrm{C}(19)-\mathrm{C}(18)-\mathrm{P}(1)$

$\mathrm{C}(20)-\mathrm{C}(18)-\mathrm{P}(1)$

$\mathrm{C}(19)-\mathrm{C}(18)-\mathrm{H}(18)$

$\mathrm{C}(20)-\mathrm{C}(18)-\mathrm{H}(18)$

$\mathrm{P}(1)-\mathrm{C}(18)-\mathrm{H}(18)$

$\mathrm{C}(18)-\mathrm{C}(19)-\mathrm{H}(19 \mathrm{~A})$

$\mathrm{C}(18)-\mathrm{C}(19)-\mathrm{H}(19 \mathrm{~B})$

$\mathrm{H}(19 \mathrm{~A})-\mathrm{C}(19)-\mathrm{H}(19 \mathrm{~B})$

$\mathrm{C}(18)-\mathrm{C}(19)-\mathrm{H}(19 \mathrm{C})$

$\mathrm{H}(19 \mathrm{~A})-\mathrm{C}(19)-\mathrm{H}(19 \mathrm{C})$

$\mathrm{H}(19 \mathrm{~B})-\mathrm{C}(19)-\mathrm{H}(19 \mathrm{C})$

$\mathrm{C}(18)-\mathrm{C}(20)-\mathrm{H}(20 \mathrm{~A})$

$\mathrm{C}(18)-\mathrm{C}(20)-\mathrm{H}(20 \mathrm{~B})$

$\mathrm{H}(20 \mathrm{~A})-\mathrm{C}(20)-\mathrm{H}(20 \mathrm{~B})$

$\mathrm{C}(18)-\mathrm{C}(20)-\mathrm{H}(20 \mathrm{C})$

$\mathrm{H}(20 \mathrm{~A})-\mathrm{C}(20)-\mathrm{H}(20 \mathrm{C})$

$\mathrm{H}(20 \mathrm{~B})-\mathrm{C}(20)-\mathrm{H}(20 \mathrm{C})$

$C(22)-C(21)-C(23)$

$\mathrm{C}(22)-\mathrm{C}(21)-\mathrm{P}(1)$

$\mathrm{C}(23)-\mathrm{C}(21)-\mathrm{P}(1)$

$\mathrm{C}(22)-\mathrm{C}(21)-\mathrm{H}(21)$

$\mathrm{C}(23)-\mathrm{C}(21)-\mathrm{H}(21)$

$\mathrm{P}(1)-\mathrm{C}(21)-\mathrm{H}(21)$
$111(3)$

$118.2(4)$

$123.8(4)$

$117.9(4)$

$121.0(4)$

119.5

119.5

$120.5(4)$

119.8

119.8

119.2 (4)

120.4

120.4

$120.3(4)$

119.9

119.9

$120.9(4)$

119.6

119.6

$121.4(4)$

$113.6(3)$

$124.9(3)$

$69.7(2)$

$127.4(3)$

$68.67(13)$

109.5

109.5

109.5

109.5

109.5

109.5

$73.8(2)$

$122(3)$

$98(3)$

119 (3)

$123(3)$

$113(4)$

$111.2(4)$

$111.3(3)$

114.7 (3)

106.4

106.4

106.4

109.5

109.5

109.5

109.5

109.5

109.5

109.5

109.5

109.5

109.5

109.5

109.5

$111.3(4)$

$112.5(3)$

$114.6(3)$

105.9

105.9

105.9 


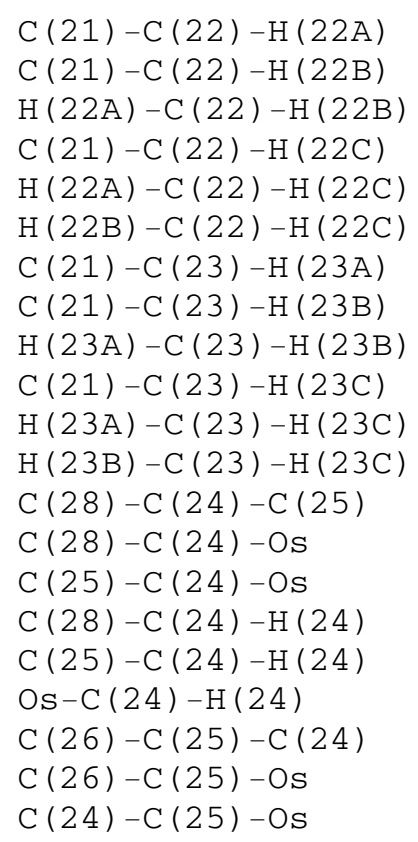

Symmetry transformations used to generate equivalent atoms: 
Table 4. Anisotropic displacement parameters ( $A^{\wedge} 2 \times 10^{\wedge} 3$ ) for 5 . The anisotropic displacement factor exponent takes the form:

$-2 \mathrm{pi}^{\wedge} 2 \mathrm{~h}$ [ $2 \mathrm{a}^{\star \wedge} 2 \mathrm{U} 11+\ldots+2 \mathrm{~h} \mathrm{k} \mathrm{a}^{*} \mathrm{~b}$ * U12 ]

\begin{tabular}{|c|c|c|c|c|c|c|}
\hline & U11 & U22 & U33 & U2 3 & U13 & U12 \\
\hline Os & $14(1)$ & $16(1)$ & $19(1)$ & $2(1)$ & $1(1)$ & $0(1)$ \\
\hline $\mathrm{P}(1)$ & $15(1)$ & $16(1)$ & $20(1)$ & $2(1)$ & $0(1)$ & $0(1)$ \\
\hline$P(2)$ & $17(1)$ & $31(1)$ & $24(1)$ & $-4(1)$ & $2(1)$ & $0(1)$ \\
\hline$F(1)$ & $57(2)$ & $29(2)$ & $42(2)$ & $-5(1)$ & $-12(2)$ & $6(1)$ \\
\hline$F(2)$ & $40(2)$ & $84(3)$ & $39(2)$ & $-2(2)$ & $19(1)$ & $-20(2)$ \\
\hline$F(3)$ & $40(2)$ & $32(2)$ & $68(2)$ & $-11(2)$ & $3(2)$ & $7(1)$ \\
\hline$F(4)$ & $24(2)$ & $53(2)$ & $32(2)$ & $6(1)$ & $7(1)$ & $-1(1)$ \\
\hline$F(5)$ & $28(2)$ & $51(2)$ & $28(1)$ & $-8(1)$ & $-6(1)$ & $-4(1)$ \\
\hline$F(6)$ & $19(1)$ & $57(2)$ & $45(2)$ & $-5(1)$ & $-11(1)$ & $1(1)$ \\
\hline$C(1)$ & $16(2)$ & $19(2)$ & $22(2)$ & $2(2)$ & $8(2)$ & $3(2)$ \\
\hline$C(2)$ & $15(2)$ & $24(2)$ & $21(2)$ & $0(2)$ & $5(2)$ & $-1(2)$ \\
\hline$C(3)$ & $21(2)$ & $20(2)$ & $20(2)$ & $3(2)$ & $0(2)$ & $1(2)$ \\
\hline$C(4)$ & $21(2)$ & $18(2)$ & $28(2)$ & $2(2)$ & $5(2)$ & $1(2)$ \\
\hline$C(5)$ & $27(2)$ & $22(2)$ & $29(2)$ & $-6(2)$ & $5(2)$ & $-1(2)$ \\
\hline$C(6)$ & $31(3)$ & $32(3)$ & $23(2)$ & $-5(2)$ & $-2(2)$ & $4(2)$ \\
\hline$C(7)$ & $28(2)$ & $24(2)$ & $22(2)$ & $4(2)$ & $2(2)$ & $4(2)$ \\
\hline$C(8)$ & $18(2)$ & $18(2)$ & $24(2)$ & $-1(2)$ & $5(2)$ & $-1(2)$ \\
\hline$C(9)$ & $19(2)$ & $13(2)$ & $29(2)$ & $-2(2)$ & $-4(2)$ & $-1(2)$ \\
\hline$C(10)$ & $22(2)$ & $26(2)$ & $30(2)$ & $-3(2)$ & $-2(2)$ & $2(2)$ \\
\hline$C(11)$ & $22(2)$ & $29(3)$ & $42(3)$ & $-10(2)$ & $-7(2)$ & $8(2)$ \\
\hline$C(12)$ & $30(3)$ & $24(2)$ & $42(3)$ & $3(2)$ & $-16(2)$ & $3(2)$ \\
\hline$C(13)$ & $34(3)$ & $33(3)$ & $29(2)$ & $6(2)$ & $-7(2)$ & $-4(2)$ \\
\hline$C(14)$ & $18(2)$ & $29(2)$ & $28(2)$ & $-3(2)$ & $-2(2)$ & $1(2)$ \\
\hline$C(15)$ & $18(2)$ & $20(2)$ & $21(2)$ & $4(2)$ & $0(2)$ & $3(2)$ \\
\hline$C(16)$ & $21(2)$ & $20(2)$ & $24(2)$ & $4(2)$ & $2(2)$ & $-1(2)$ \\
\hline$C(17)$ & $24(2)$ & $20(2)$ & $20(2)$ & $4(2)$ & $-2(2)$ & $0(2)$ \\
\hline$C(18)$ & $15(2)$ & $21(2)$ & $21(2)$ & $-2(2)$ & $0(2)$ & $-1(2)$ \\
\hline C (19) & 27 (3) & 37 ( 3 ) & 31 (3) & $5(2)$ & $-12(2)$ & $-4(2)$ \\
\hline$C(20)$ & $30(3)$ & $24(3)$ & $45(3)$ & $5(2)$ & $-7(2)$ & $-9(2)$ \\
\hline$C(21)$ & $18(2)$ & $17(2)$ & $27(2)$ & $6(2)$ & $4(2)$ & $4(2)$ \\
\hline$C(22)$ & $33(3)$ & $42(3)$ & $30(3)$ & $3(2)$ & $11(2)$ & $11(2)$ \\
\hline$C(23)$ & $29(3)$ & $18(2)$ & $42(3)$ & $1(2)$ & $1(2)$ & $6(2)$ \\
\hline$C(24)$ & $22(2)$ & $24(2)$ & $21(2)$ & $2(2)$ & $4(2)$ & $-3(2)$ \\
\hline$C(25)$ & $20(2)$ & $20(2)$ & 31 (2) & $5(2)$ & $8(2)$ & 1 (2) \\
\hline$C(26)$ & $17(2)$ & $27(2)$ & $27(2)$ & $5(2)$ & $1(2)$ & $-4(2)$ \\
\hline$C(27)$ & $23(2)$ & $23(2)$ & $27(2)$ & $3(2)$ & $3(2)$ & $-7(2)$ \\
\hline$C(28)$ & $22(2)$ & $17(2)$ & $27(2)$ & $4(2)$ & $3(2)$ & $-2(2)$ \\
\hline
\end{tabular}


Table 5. Hydrogen coordinates ( $\left.\mathrm{x} 10^{\wedge} 4\right)$ and isotropic displacement parameters $\left(A^{\wedge} 2 \times 10^{\wedge} 3\right)$ for 5 .

\begin{tabular}{|c|c|c|c|c|}
\hline & $\mathrm{x}$ & $\mathrm{Y}$ & $\mathrm{z}$ & $\mathrm{U}$ (eq) \\
\hline $\mathrm{H}(1)$ & $1880(40)$ & $1880(20)$ & $8000(40)$ & 23 \\
\hline $\mathrm{H}(3)$ & 2494 & 355 & 9388 & 24 \\
\hline $\mathrm{H}(4)$ & 2144 & -667 & 8607 & 27 \\
\hline $\mathrm{H}(5)$ & 1161 & -764 & 6932 & 31 \\
\hline $\mathrm{H}(6)$ & 560 & 195 & 6001 & 34 \\
\hline $\mathrm{H}(7)$ & 914 & 1225 & 6763 & 29 \\
\hline $\mathrm{H}(8)$ & $2880(40)$ & $2190(20)$ & $9430(30)$ & 23 \\
\hline $\mathrm{H}(10)$ & 4498 & 1201 & 9251 & 31 \\
\hline $\mathrm{H}(11)$ & 5724 & 568 & 10386 & 37 \\
\hline $\mathrm{H}(12)$ & 5226 & 377 & 12171 & 39 \\
\hline $\mathrm{H}(13)$ & 3515 & 858 & 12822 & 39 \\
\hline $\mathrm{H}(14)$ & 2297 & 1510 & 11688 & 30 \\
\hline $\mathrm{H}(16 \mathrm{~A})$ & 1677 & 2879 & 7356 & 32 \\
\hline $\mathrm{H}(16 \mathrm{~B})$ & 2569 & 3024 & 8370 & 32 \\
\hline$H(16 C)$ & 1753 & 3604 & 7869 & 32 \\
\hline $\mathrm{H}(17 \mathrm{~A})$ & $-960(40)$ & $2710(20)$ & $8740(30)$ & 26 \\
\hline $\mathrm{H}(17 \mathrm{~B})$ & $-300(40)$ & $2520(20)$ & $7740(40)$ & 26 \\
\hline $\mathrm{H}(18)$ & 2961 & 3210 & 10204 & 23 \\
\hline $\mathrm{H}(19 \mathrm{~A})$ & 3486 & 3389 & 11996 & 48 \\
\hline $\mathrm{H}(19 \mathrm{~B})$ & 2579 & 2780 & 11928 & 48 \\
\hline$H(19 C)$ & 2128 & 3493 & 12292 & 48 \\
\hline $\mathrm{H}(20 \mathrm{~A})$ & 1975 & 4408 & 10958 & 50 \\
\hline $\mathrm{H}(20 \mathrm{~B})$ & 2169 & 4268 & 9704 & 50 \\
\hline $\mathrm{H}(20 \mathrm{C})$ & 3289 & 4301 & 10542 & 50 \\
\hline $\mathrm{H}(21)$ & -1085 & 3262 & 10409 & 25 \\
\hline $\mathrm{H}(22 \mathrm{~A})$ & 179 & 3769 & 12266 & 52 \\
\hline$H(22 B)$ & -285 & 3023 & 12183 & 52 \\
\hline $\mathrm{H}(22 \mathrm{C})$ & -1216 & 3621 & 12150 & 52 \\
\hline $\mathrm{H}(23 \mathrm{~A})$ & -1323 & 4374 & 10395 & 45 \\
\hline$H(23 B)$ & -357 & 4234 & 9509 & 45 \\
\hline $\mathrm{H}(23 \mathrm{C})$ & 56 & 4508 & 10679 & 45 \\
\hline $\mathrm{H}(24)$ & 358 & 1641 & 11861 & 27 \\
\hline $\mathrm{H}(25)$ & -1434 & 2238 & 10954 & 28 \\
\hline $\mathrm{H}(26)$ & -1940 & 1654 & 9191 & 29 \\
\hline $\mathrm{H}(27)$ & -438 & 754 & 8923 & 29 \\
\hline $\mathrm{H}(28)$ & 973 & 728 & 10578 & 26 \\
\hline
\end{tabular}


Table 6. Crystal data and structure refinement for 8 .

\begin{tabular}{|c|c|}
\hline Identification code & 8 \\
\hline Empirical formula & C29 H39 Os P \\
\hline Formula weight & 608.77 \\
\hline Temperature & $100(2) \mathrm{K}$ \\
\hline Wavelength & $0.71073 \mathrm{~A}$ \\
\hline Crystal system, space group & Monoclinic, $\quad$ P $121 / \mathrm{n} 1$ \\
\hline Unit cell dimensions & $\begin{array}{lll}\mathrm{a}=10.0637(5) \mathrm{A} & \text { alpha }=90 \text { deg. } \\
\mathrm{b}=18.3653(9) & \mathrm{A} & \text { beta }=90.5290(10) \mathrm{deg} . \\
\mathrm{c}=13.7078(7) \mathrm{A} & \text { gamma }=90 \mathrm{deg} .\end{array}$ \\
\hline Volume & $2533.4(2) \quad A^{\wedge} 3$ \\
\hline Z, Calculated density & 4, $1.596 \mathrm{Mg} / \mathrm{m}^{\wedge} 3$ \\
\hline Absorption coefficient & $5.111 \mathrm{~mm}^{\wedge}-1$ \\
\hline$F(000)$ & 1216 \\
\hline Crystal size & $0.16 \times 0.12 \times 0.08 \mathrm{~mm}$ \\
\hline Theta range for data collection & 1.85 to $28.60 \mathrm{deg}$. \\
\hline Limiting indices & $-12<=\mathrm{h}<=13, \quad-23<=\mathrm{k}<=24, \quad-18<=1<=18$ \\
\hline Reflections collected / unique & $30444 / 6065[R($ int $)=0.0453]$ \\
\hline Completeness to theta $=28.60$ & $93.4 \%$ \\
\hline Absorption correction & Semi-empirical from equivalents \\
\hline Max. and min. transmission & 0.647 and 0.533 \\
\hline Refinement method & Full-matrix least-squares on $\mathrm{F}^{\wedge} 2$ \\
\hline Data / restraints / parameters & $6065 / 14 / 288$ \\
\hline Goodness-of-fit on $\mathrm{F}^{\wedge} 2$ & 1.015 \\
\hline Final $R$ indices [I>2sigma(I)] & $\mathrm{R} 1=0.0326, \mathrm{wR} 2=0.0690$ \\
\hline R indices (all data) & $\mathrm{R} 1=0.0444, \mathrm{wR} 2=0.0723$ \\
\hline Largest diff. peak and hole & 1.741 and -1.632 e. $A^{\wedge}-3$ \\
\hline
\end{tabular}


Table 7. Atomic coordinates $\left(x 0^{\wedge} 4\right)$ and equivalent isotropic displacement parameters $\left(A^{\wedge} 2 \times 10^{\wedge} 3\right)$ for 8 .

$\mathrm{U}(\mathrm{eq})$ is defined as one third of the trace of the orthogonalized Uij tensor.

\begin{tabular}{|c|c|c|c|c|}
\hline & $\mathrm{x}$ & $\mathrm{Y}$ & $z$ & $\mathrm{U}$ (eq) \\
\hline Os & $4090(1)$ & $1917(1)$ & $9819(1)$ & $18(1)$ \\
\hline $\mathrm{P}$ & $1830(1)$ & $1884(1)$ & $9621(1)$ & $19(1)$ \\
\hline$C(1)$ & $4284(4)$ & $2611(2)$ & $8533(3)$ & $17(1)$ \\
\hline$C(2)$ & $5040(4)$ & $3301(2)$ & $8640(3)$ & $20(1)$ \\
\hline$C(3)$ & $6429(5)$ & $3337(3)$ & $8609(3)$ & $24(1)$ \\
\hline$C(4)$ & $7093(5)$ & 3988 ( 3 ) & 8697 ( 3 ) & $31(1)$ \\
\hline$C(5)$ & $6405(5)$ & $4626(3)$ & $8806(3)$ & $33(1)$ \\
\hline$C(6)$ & $5041(5)$ & $4610(3)$ & $8821(3)$ & $31(1)$ \\
\hline$C(7)$ & $4365(5)$ & $3957(2)$ & $8737(3)$ & $25(1)$ \\
\hline$C(8)$ & $4900(4)$ & $1917(2)$ & $8373(3)$ & $19(1)$ \\
\hline$C(9)$ & $4480(4)$ & $1394(2)$ & $7605(3)$ & $20(1)$ \\
\hline$C(10)$ & $5119(4)$ & $716(2)$ & $7542(3)$ & $25(1)$ \\
\hline $\mathrm{C}(11)$ & $4765(5)$ & $216(3)$ & $6844(4)$ & $30(1)$ \\
\hline$C(12)$ & $3784(5)$ & $372(3)$ & $6166(4)$ & $30(1)$ \\
\hline$C(13)$ & $3148(5)$ & $1039(3)$ & $6202(3)$ & $26(1)$ \\
\hline$C(14)$ & $3510(4)$ & $1542(2)$ & $6908(3)$ & $21(1)$ \\
\hline$C(15)$ & $5595(5)$ & $1500(3)$ & $10874(3)$ & $27(1)$ \\
\hline$C(16)$ & $6355(5)$ & $805(3)$ & $10804(4)$ & $42(1)$ \\
\hline$C(17)$ & $6023(5)$ & $2195(3)$ & $10568(3)$ & $26(1)$ \\
\hline$C(18)$ & $5080(5)$ & $2725(3)$ & $10862(3)$ & $31(1)$ \\
\hline$C(19)$ & $4062(5)$ & $2362(3)$ & $11357(3)$ & $32(1)$ \\
\hline$C(20)$ & 4365 ( 5) & $1600(3)$ & $11357(3)$ & $30(1)$ \\
\hline$C(21 A)$ & $1224(12)$ & $2825(5)$ & $9480(6)$ & $24(3)$ \\
\hline$C(22 \mathrm{~A})$ & 745 (12) & $3115(6)$ & $8652(8)$ & $25(3)$ \\
\hline$C(23 A)$ & $1474(12)$ & $3294(6)$ & 10406 ( 8) & $30(3)$ \\
\hline$C(24 B)$ & $861(19)$ & $2764(7)$ & $9564(9)$ & $27(4)$ \\
\hline$C(25 B)$ & $800(20)$ & $3026(12)$ & $8489(11)$ & $35(6)$ \\
\hline$C(26 B)$ & $1404(17)$ & 3377 ( 8 ) & $10226(12)$ & $19(4)$ \\
\hline$C(21 B)$ & $998(13)$ & $1404(9)$ & $10684(11)$ & $23(4)$ \\
\hline$C(22 B)$ & $-48(17)$ & $1676(9)$ & $11171(14)$ & $41(5)$ \\
\hline$C(23 B)$ & $1574(18)$ & $642(7)$ & $10880(11)$ & $30(4)$ \\
\hline$C(24 A)$ & $1039(8)$ & $1582(6)$ & $10753(7)$ & $22(2)$ \\
\hline$C(25 A)$ & $-446(8)$ & $1789(5)$ & $10877(7)$ & $28(3)$ \\
\hline$C(26 A)$ & $1216(10)$ & $765(5)$ & $10953(6)$ & $20(2)$ \\
\hline$C(27)$ & $1047(4)$ & $1393(2)$ & $8584(3)$ & $23(1)$ \\
\hline $\mathrm{C}(28)$ & $1597(5)$ & $613(2)$ & $8452(3)$ & $26(1)$ \\
\hline$C(29)$ & $-470(5)$ & $1374(3)$ & $8562(4)$ & $30(1)$ \\
\hline
\end{tabular}


Table 8. Bond lengths [A] and angles [deg] for 8 .

\begin{tabular}{|c|c|c|c|}
\hline $\mathrm{Os}-\mathrm{C}(8)$ & $2.150(4)$ & $C(21 B)-C(22 B)$ & $1.348(7)$ \\
\hline $\mathrm{Os}-\mathrm{C}(1)$ & $2.186(4)$ & $C(21 B)-C(23 B)$ & $1.537(7)$ \\
\hline $\mathrm{Os}-\mathrm{C}(20)$ & $2.201(5)$ & $C(24 A)-C(26 A)$ & $1.536(7)$ \\
\hline $\mathrm{Os}-\mathrm{C}(15)$ & $2.220(5)$ & $C(24 A)-C(25 A)$ & $1.552(8)$ \\
\hline $\mathrm{Os}-\mathrm{C}(17)$ & $2.249(4)$ & $C(27)-C(29)$ & $1.527(6)$ \\
\hline Os -C (19) & $2.261(5)$ & $C(27)-C(28)$ & $1.546(6)$ \\
\hline $\mathrm{Os}-\mathrm{C}(18)$ & $2.283(5)$ & $\mathrm{C}(27)-\mathrm{H}(27)$ & 1.0000 \\
\hline $\mathrm{Os}-\mathrm{P}$ & $2.2886(11)$ & $\mathrm{C}(28)-\mathrm{H}(28 \mathrm{~A})$ & 0.9800 \\
\hline $\mathrm{Os}-\mathrm{H}(01)$ & $1.55(6)$ & $\mathrm{C}(28)-\mathrm{H}(28 \mathrm{~B})$ & 0.9800 \\
\hline$P-C(24 A)$ & $1.836(9)$ & $\mathrm{C}(28)-\mathrm{H}(28 \mathrm{C})$ & 0.9800 \\
\hline$P-C(21 A)$ & $1.842(9)$ & $\mathrm{C}(29)-\mathrm{H}(29 A)$ & 0.9800 \\
\hline$P-C(27)$ & $1.853(4)$ & $\mathrm{C}(29)-\mathrm{H}(29 \mathrm{~B})$ & 0.9800 \\
\hline$P-C(24 B)$ & $1.888(15)$ & $\mathrm{C}(29)-\mathrm{H}(29 \mathrm{C})$ & 0.9800 \\
\hline$P-C(21 B)$ & $1.904(14)$ & & \\
\hline$C(1)-C(8)$ & $1.434(6)$ & $\mathrm{C}(8)-\mathrm{Os}-\mathrm{C}(1)$ & $38.62(15)$ \\
\hline$C(1)-C(2)$ & $1.485(6)$ & $C(8)-O s-C(20)$ & $146.85(18)$ \\
\hline $\mathrm{C}(1)-\mathrm{H}(1)$ & $0.98(4)$ & $C(1)-O s-C(20)$ & $156.19(18)$ \\
\hline$C(2)-C(7)$ & $1.391(6)$ & $C(8)-O s-C(15)$ & $109.74(18)$ \\
\hline$C(2)-C(3)$ & $1.400(6)$ & $C(1)-O s-C(15)$ & $131.33(17)$ \\
\hline$C(3)-C(4)$ & $1.375(6)$ & $C(20)-O s-C(15)$ & $37.50(18)$ \\
\hline $\mathrm{C}(3)-\mathrm{H}(3)$ & 0.9500 & $\mathrm{C}(8)-\mathrm{Os}-\mathrm{C}(17)$ & $94.98(18)$ \\
\hline$C(4)-C(5)$ & $1.370(7)$ & $C(1)-O s-C(17)$ & $98.77(17)$ \\
\hline $\mathrm{C}(4)-\mathrm{H}(4)$ & 0.9500 & $C(20)-O s-C(17)$ & $61.47(18)$ \\
\hline$C(5)-C(6)$ & $1.373(7)$ & $C(15)-O s-C(17)$ & $36.78(16)$ \\
\hline $\mathrm{C}(5)-\mathrm{H}(5)$ & 0.9500 & $\mathrm{C}(8)-\mathrm{Os}-\mathrm{C}(19)$ & $150.14(18)$ \\
\hline$C(6)-C(7)$ & $1.382(6)$ & $C(1)-O s-C(19)$ & $122.92(18)$ \\
\hline $\mathrm{C}(6)-\mathrm{H}(6)$ & 0.9500 & $C(20)-O s-C(19)$ & $37.37(18)$ \\
\hline $\mathrm{C}(7)-\mathrm{H}(7)$ & 0.9500 & $C(15)-O s-C(19)$ & $62.08(18)$ \\
\hline$C(8)-C(9)$ & $1.484(6)$ & $C(17)-O s-C(19)$ & $60.71(18)$ \\
\hline $\mathrm{C}(8)-\mathrm{H}(8)$ & $0.92(5)$ & $\mathrm{C}(8)-\mathrm{Os}-\mathrm{C}(18)$ & $114.22(18)$ \\
\hline$C(9)-C(14)$ & $1.386(6)$ & $\mathrm{C}(1)-\mathrm{Os}-\mathrm{C}(18)$ & $94.86(17)$ \\
\hline$C(9)-C(10)$ & $1.405(6)$ & $C(20)-O s-C(18)$ & $61.42(18)$ \\
\hline$C(10)-C(11)$ & $1.371(6)$ & $C(15)-O s-C(18)$ & $61.64(17)$ \\
\hline $\mathrm{C}(10)-\mathrm{H}(10)$ & 0.9500 & $\mathrm{C}(17)-\mathrm{Os}-\mathrm{C}(18)$ & $36.53(17)$ \\
\hline$C(11)-C(12)$ & $1.380(7)$ & $\mathrm{C}(19)-\mathrm{Os}-\mathrm{C}(18)$ & $35.93(17)$ \\
\hline $\mathrm{C}(11)-\mathrm{H}(11)$ & 0.9500 & $C(8)-O s-P$ & $105.99(12)$ \\
\hline$C(12)-C(13)$ & $1.383(6)$ & $C(1)-O s-P$ & $90.90(12)$ \\
\hline $\mathrm{C}(12)-\mathrm{H}(12)$ & 0.9500 & $C(20)-O S-P$ & $102.87(14)$ \\
\hline$C(13)-C(14)$ & $1.385(6)$ & $C(15)-O S-P$ & $137.70(13)$ \\
\hline $\mathrm{C}(13)-\mathrm{H}(13)$ & 0.9500 & $C(17)-O S-P$ & $156.09(13)$ \\
\hline $\mathrm{C}(14)-\mathrm{H}(14)$ & 0.9500 & $C(19)-O S-P$ & $95.70(13)$ \\
\hline$C(15)-C(17)$ & $1.411(6)$ & $\mathrm{C}(18)-\mathrm{Os}-\mathrm{P}$ & $121.24(14)$ \\
\hline$C(15)-C(20)$ & $1.422(7)$ & $\mathrm{C}(8)-\mathrm{Os}-\mathrm{H}(01)$ & $82(2)$ \\
\hline$C(15)-C(16)$ & $1.491(7)$ & $\mathrm{C}(1)-\mathrm{Os}-\mathrm{H}(01)$ & $111(2)$ \\
\hline $\mathrm{C}(16)-\mathrm{H}(16 \mathrm{~A})$ & 0.9800 & $\mathrm{C}(20)-\mathrm{Os}-\mathrm{H}(01)$ & $92(2)$ \\
\hline$C(16)-H(16 B)$ & 0.9800 & $\mathrm{C}(15)-\mathrm{Os}-\mathrm{H}(01)$ & $93(2)$ \\
\hline $\mathrm{C}(16)-\mathrm{H}(16 \mathrm{C})$ & 0.9800 & $\mathrm{C}(17)-\mathrm{Os}-\mathrm{H}(01)$ & $125(2)$ \\
\hline$C(17)-C(18)$ & $1.421(7)$ & $\mathrm{C}(19)-\mathrm{Os}-\mathrm{H}(01)$ & $125(2)$ \\
\hline $\mathrm{C}(17)-\mathrm{H}(17)$ & 0.9500 & $\mathrm{C}(18)-\mathrm{Os}-\mathrm{H}(01)$ & $152(2)$ \\
\hline$C(18)-C(19)$ & $1.402(7)$ & $\mathrm{P}-\mathrm{OS}-\mathrm{H}(01)$ & $70(2)$ \\
\hline $\mathrm{C}(18)-\mathrm{H}(18)$ & 0.9500 & $C(24 A)-P-C(21 A)$ & $103.1(4)$ \\
\hline$C(19)-C(20)$ & $1.431(7)$ & $C(24 A)-P-C(27)$ & $108.5(4)$ \\
\hline $\mathrm{C}(19)-\mathrm{H}(19)$ & 0.9500 & $C(21 A)-P-C(27)$ & $103.8(3)$ \\
\hline $\mathrm{C}(20)-\mathrm{H}(20)$ & 0.9500 & $C(24 A)-P-C(24 B)$ & $93.7(5)$ \\
\hline$C(21 A)-C(22 A)$ & $1.340(7)$ & $C(21 A)-P-C(24 B)$ & $12.3(5)$ \\
\hline$C(21 A)-C(23 A)$ & $1.551(7)$ & $C(27)-P-C(24 B)$ & $99.7(4)$ \\
\hline$C(24 B)-C(26 B)$ & $1.543(8)$ & $C(24 A)-P-C(21 B)$ & $10.3(6)$ \\
\hline$C(24 B)-C(25 B)$ & $1.552(9)$ & $C(21 A)-P-C(21 B)$ & $111.6(5)$ \\
\hline
\end{tabular}




\begin{tabular}{|c|c|}
\hline $\mathrm{C}(27)-\mathrm{P}-\mathrm{C}(21 \mathrm{~B})$ & $100.1(6)$ \\
\hline$C(24 B)-P-C(21 B)$ & $101.4(6)$ \\
\hline$C(24 A)-P-O S$ & $110.2(3)$ \\
\hline$C(21 A)-P-O S$ & $108.4(4)$ \\
\hline $\mathrm{C}(27)-\mathrm{P}-\mathrm{Os}$ & $121.24(15)$ \\
\hline$C(24 B)-P-O S$ & $119.7(5)$ \\
\hline $\mathrm{C}(21 \mathrm{~B})-\mathrm{P}-\mathrm{OS}$ & $111.4(4)$ \\
\hline$C(8)-C(1)-C(2)$ & $123.5(4)$ \\
\hline$C(8)-C(1)-O S$ & $69.4(2)$ \\
\hline$C(2)-C(1)-O s$ & $117.9(3)$ \\
\hline $\mathrm{C}(8)-\mathrm{C}(1)-\mathrm{H}(1)$ & $116(3)$ \\
\hline $\mathrm{C}(2)-\mathrm{C}(1)-\mathrm{H}(1)$ & $111(3)$ \\
\hline $\mathrm{Os}-\mathrm{C}(1)-\mathrm{H}(1)$ & $113(3)$ \\
\hline$C(7)-C(2)-C(3)$ & $116.8(4)$ \\
\hline$C(7)-C(2)-C(1)$ & $120.0(4)$ \\
\hline$C(3)-C(2)-C(1)$ & $123.2(4)$ \\
\hline$C(4)-C(3)-C(2)$ & $121.5(5)$ \\
\hline $\mathrm{C}(4)-\mathrm{C}(3)-\mathrm{H}(3)$ & 119.2 \\
\hline $\mathrm{C}(2)-\mathrm{C}(3)-\mathrm{H}(3)$ & 119.2 \\
\hline$C(5)-C(4)-C(3)$ & $120.5(5)$ \\
\hline $\mathrm{C}(5)-\mathrm{C}(4)-\mathrm{H}(4)$ & 119.7 \\
\hline $\mathrm{C}(3)-\mathrm{C}(4)-\mathrm{H}(4)$ & 119.7 \\
\hline$C(4)-C(5)-C(6)$ & $119.3(4)$ \\
\hline $\mathrm{C}(4)-\mathrm{C}(5)-\mathrm{H}(5)$ & 120.4 \\
\hline $\mathrm{C}(6)-\mathrm{C}(5)-\mathrm{H}(5)$ & 120.4 \\
\hline$C(5)-C(6)-C(7)$ & $120.6(5)$ \\
\hline $\mathrm{C}(5)-\mathrm{C}(6)-\mathrm{H}(6)$ & 119.7 \\
\hline $\mathrm{C}(7)-\mathrm{C}(6)-\mathrm{H}(6)$ & 119.7 \\
\hline$C(6)-C(7)-C(2)$ & $121.3(5)$ \\
\hline $\mathrm{C}(6)-\mathrm{C}(7)-\mathrm{H}(7)$ & 119.4 \\
\hline $\mathrm{C}(2)-\mathrm{C}(7)-\mathrm{H}(7)$ & 119.4 \\
\hline$C(1)-C(8)-C(9)$ & $124.2(4)$ \\
\hline $\mathrm{C}(1)-\mathrm{C}(8)-\mathrm{OS}$ & $72.0(2)$ \\
\hline $\mathrm{C}(9)-\mathrm{C}(8)-\mathrm{Os}$ & $123.1(3)$ \\
\hline $\mathrm{C}(1)-\mathrm{C}(8)-\mathrm{H}(8)$ & $108(3)$ \\
\hline $\mathrm{C}(9)-\mathrm{C}(8)-\mathrm{H}(8)$ & $116(3)$ \\
\hline $\mathrm{Os}-\mathrm{C}(8)-\mathrm{H}(8)$ & $104(3)$ \\
\hline$C(14)-C(9)-C(10)$ & $116.8(4)$ \\
\hline$C(14)-C(9)-C(8)$ & $123.8(4)$ \\
\hline$C(10)-C(9)-C(8)$ & $119.3(4)$ \\
\hline$C(11)-C(10)-C(9)$ & $121.4(4)$ \\
\hline $\mathrm{C}(11)-\mathrm{C}(10)-\mathrm{H}(10)$ & 119.3 \\
\hline $\mathrm{C}(9)-\mathrm{C}(10)-\mathrm{H}(10)$ & 119.3 \\
\hline$C(10)-C(11)-C(12)$ & $120.7(4)$ \\
\hline $\mathrm{C}(10)-\mathrm{C}(11)-\mathrm{H}(11)$ & 119.7 \\
\hline $\mathrm{C}(12)-\mathrm{C}(11)-\mathrm{H}(11)$ & 119.7 \\
\hline$C(11)-C(12)-C(13)$ & $119.3(4)$ \\
\hline $\mathrm{C}(11)-\mathrm{C}(12)-\mathrm{H}(12)$ & 120.4 \\
\hline $\mathrm{C}(13)-\mathrm{C}(12)-\mathrm{H}(12)$ & 120.4 \\
\hline$C(12)-C(13)-C(14)$ & $119.8(5)$ \\
\hline $\mathrm{C}(12)-\mathrm{C}(13)-\mathrm{H}(13)$ & 120.1 \\
\hline $\mathrm{C}(14)-\mathrm{C}(13)-\mathrm{H}(13)$ & 120.1 \\
\hline$C(13)-C(14)-C(9)$ & $122.0(4)$ \\
\hline $\mathrm{C}(13)-\mathrm{C}(14)-\mathrm{H}(14)$ & 119.0 \\
\hline $\mathrm{C}(9)-\mathrm{C}(14)-\mathrm{H}(14)$ & 119.0 \\
\hline$C(17)-C(15)-C(20)$ & $106.9(4)$ \\
\hline$C(17)-C(15)-C(16)$ & $126.7(5)$ \\
\hline$C(20)-C(15)-C(16)$ & $126.1(5)$ \\
\hline$C(17)-C(15)-O s$ & $72.7(3)$ \\
\hline$C(20)-C(15)-O s$ & $70.5(3)$ \\
\hline$C(16)-C(15)-O s$ & $126.9(3)$ \\
\hline
\end{tabular}

\begin{tabular}{|c|c|}
\hline $\mathrm{C}(15)-\mathrm{C}(16)-\mathrm{H}(16 \mathrm{~A})$ & 109.5 \\
\hline $\mathrm{C}(15)-\mathrm{C}(16)-\mathrm{H}(16 \mathrm{~B})$ & 109.5 \\
\hline $\mathrm{H}(16 \mathrm{~A})-\mathrm{C}(16)-\mathrm{H}(16 \mathrm{~B})$ & 109.5 \\
\hline $\mathrm{C}(15)-\mathrm{C}(16)-\mathrm{H}(16 \mathrm{C})$ & 109.5 \\
\hline $\mathrm{H}(16 \mathrm{~A})-\mathrm{C}(16)-\mathrm{H}(16 \mathrm{C})$ & 109.5 \\
\hline $\mathrm{H}(16 \mathrm{~B})-\mathrm{C}(16)-\mathrm{H}(16 \mathrm{C})$ & 109.5 \\
\hline$C(15)-C(17)-C(18)$ & $109.2(4)$ \\
\hline $\mathrm{C}(15)-\mathrm{C}(17)-\mathrm{Os}$ & $70.5(3)$ \\
\hline $\mathrm{C}(18)-\mathrm{C}(17)-\mathrm{Os}$ & $73.0(3)$ \\
\hline $\mathrm{C}(15)-\mathrm{C}(17)-\mathrm{H}(17)$ & 125.4 \\
\hline $\mathrm{C}(18)-\mathrm{C}(17)-\mathrm{H}(17)$ & 125.4 \\
\hline $\mathrm{Os}-\mathrm{C}(17)-\mathrm{H}(17)$ & 122.7 \\
\hline$C(19)-C(18)-C(17)$ & $107.7(4)$ \\
\hline $\mathrm{C}(19)-\mathrm{C}(18)-\mathrm{Os}$ & $71.2(3)$ \\
\hline $\mathrm{C}(17)-\mathrm{C}(18)-\mathrm{Os}$ & $70.4(3)$ \\
\hline $\mathrm{C}(19)-\mathrm{C}(18)-\mathrm{H}(18)$ & 126.2 \\
\hline $\mathrm{C}(17)-\mathrm{C}(18)-\mathrm{H}(18)$ & 126.2 \\
\hline $\mathrm{Os}-\mathrm{C}(18)-\mathrm{H}(18)$ & 123.9 \\
\hline$C(18)-C(19)-C(20)$ & $108.0(5)$ \\
\hline $\mathrm{C}(18)-\mathrm{C}(19)-\mathrm{Os}$ & $72.9(3)$ \\
\hline $\mathrm{C}(20)-\mathrm{C}(19)-\mathrm{Os}$ & $69.1(3)$ \\
\hline $\mathrm{C}(18)-\mathrm{C}(19)-\mathrm{H}(19)$ & 126.0 \\
\hline $\mathrm{C}(20)-\mathrm{C}(19)-\mathrm{H}(19)$ & 126.0 \\
\hline $\mathrm{Os}-\mathrm{C}(19)-\mathrm{H}(19)$ & 123.6 \\
\hline$C(15)-C(20)-C(19)$ & $108.2(4)$ \\
\hline$C(15)-C(20)-O S$ & $72.0(3)$ \\
\hline$C(19)-C(20)-O s$ & $73.6(3)$ \\
\hline $\mathrm{C}(15)-\mathrm{C}(20)-\mathrm{H}(20)$ & 125.9 \\
\hline $\mathrm{C}(19)-\mathrm{C}(20)-\mathrm{H}(20)$ & 125.9 \\
\hline $\mathrm{Os}-\mathrm{C}(20)-\mathrm{H}(20)$ & 120.3 \\
\hline$C(22 A)-C(21 A)-C(23 A)$ & $121.8(9)$ \\
\hline$C(22 A)-C(21 A)-P$ & $125.3(8)$ \\
\hline$C(23 A)-C(21 A)-P$ & $112.6(6)$ \\
\hline$C(26 B)-C(24 B)-C(25 B)$ & $110.1(13)$ \\
\hline$C(26 B)-C(24 B)-P$ & $114.8(11)$ \\
\hline$C(25 B)-C(24 B)-P$ & $108.8(12)$ \\
\hline$C(22 B)-C(21 B)-C(23 B)$ & $123.1(12)$ \\
\hline$C(22 B)-C(21 B)-P$ & $123.9(10)$ \\
\hline$C(23 B)-C(21 B)-P$ & $112.8(8)$ \\
\hline$C(26 A)-C(24 A)-C(25 A)$ & $109.2(7)$ \\
\hline$C(26 A)-C(24 A)-P$ & $113.3(6)$ \\
\hline$C(25 A)-C(24 A)-P$ & $116.4(6)$ \\
\hline$C(29)-C(27)-C(28)$ & $109.6(4)$ \\
\hline$C(29)-C(27)-P$ & $116.4(3)$ \\
\hline$C(28)-C(27)-P$ & $113.0(3)$ \\
\hline $\mathrm{C}(29)-\mathrm{C}(27)-\mathrm{H}(27)$ & 105.7 \\
\hline $\mathrm{C}(28)-\mathrm{C}(27)-\mathrm{H}(27)$ & 105.7 \\
\hline $\mathrm{P}-\mathrm{C}(27)-\mathrm{H}(27)$ & 105.7 \\
\hline $\mathrm{C}(27)-\mathrm{C}(28)-\mathrm{H}(28 \mathrm{~A})$ & 109.5 \\
\hline $\mathrm{C}(27)-\mathrm{C}(28)-\mathrm{H}(28 \mathrm{~B})$ & 109.5 \\
\hline $\mathrm{H}(28 \mathrm{~A})-\mathrm{C}(28)-\mathrm{H}(28 \mathrm{~B})$ & 109.5 \\
\hline $\mathrm{C}(27)-\mathrm{C}(28)-\mathrm{H}(28 \mathrm{C})$ & 109.5 \\
\hline $\mathrm{H}(28 \mathrm{~A})-\mathrm{C}(28)-\mathrm{H}(28 \mathrm{C})$ & 109.5 \\
\hline $\mathrm{H}(28 \mathrm{~B})-\mathrm{C}(28)-\mathrm{H}(28 \mathrm{C})$ & 109.5 \\
\hline $\mathrm{C}(27)-\mathrm{C}(29)-\mathrm{H}(29 \mathrm{~A})$ & 109.5 \\
\hline $\mathrm{C}(27)-\mathrm{C}(29)-\mathrm{H}(29 \mathrm{~B})$ & 109.5 \\
\hline $\mathrm{H}(29 \mathrm{~A})-\mathrm{C}(29)-\mathrm{H}(29 \mathrm{~B})$ & 109.5 \\
\hline$C(27)-C(29)-H(29 C)$ & 109.5 \\
\hline $\mathrm{H}(29 \mathrm{~A})-\mathrm{C}(29)-\mathrm{H}(29 \mathrm{C})$ & 109.5 \\
\hline $\mathrm{H}(29 \mathrm{~B})-\mathrm{C}(29)-\mathrm{H}(29 \mathrm{C})$ & 109.5 \\
\hline
\end{tabular}


Symmetry transformations used to generate equivalent atoms: 
Table 9. Anisotropic displacement parameters ( $A^{\wedge} 2 \times 10^{\wedge} 3$ ) for 8 . The anisotropic displacement factor exponent takes the form:

$-2 \mathrm{pi}^{\wedge} 2\left[\mathrm{~h}^{\wedge} 2 \mathrm{a}^{\star \wedge} 2 \mathrm{U} 11+\ldots+2 \mathrm{~h} k \mathrm{a}^{*} \mathrm{~b}\right.$ * $\left.\mathrm{U} 12\right]$

\begin{tabular}{|c|c|c|c|c|c|c|}
\hline & U11 & U2 2 & U33 & $\mathrm{U} 23$ & U13 & U12 \\
\hline Os & $19(1)$ & $19(1)$ & 17 (1) & $1(1)$ & $-1(1)$ & $-2(1)$ \\
\hline $\mathrm{P}$ & 19 (1) & $19(1)$ & $18(1)$ & $-1(1)$ & $2(1)$ & $0(1)$ \\
\hline$C(1)$ & $19(2)$ & $16(2)$ & $16(2)$ & $3(2)$ & $1(2)$ & $-1(2)$ \\
\hline$C(2)$ & $25(2)$ & $19(2)$ & $17(2)$ & $1(2)$ & $-2(2)$ & $-1(2)$ \\
\hline$C(3)$ & $26(3)$ & $24(2)$ & $23(2)$ & $2(2)$ & $4(2)$ & $-2(2)$ \\
\hline$C(4)$ & $31(3)$ & $38(3)$ & $24(3)$ & $5(2)$ & $-2(2)$ & $-16(2)$ \\
\hline$C(5)$ & $52(3)$ & $26(3)$ & $20(3)$ & $3(2)$ & $-3(2)$ & $-20(2)$ \\
\hline$C(6)$ & $53(3)$ & $17(2)$ & $23(3)$ & $-1(2)$ & $-1(2)$ & $-2(2)$ \\
\hline$C(7)$ & $33(3)$ & $22(2)$ & $20(2)$ & $0(2)$ & $3(2)$ & $-2(2)$ \\
\hline$C(8)$ & $17(2)$ & $21(2)$ & $18(2)$ & $0(2)$ & $3(2)$ & $3(2)$ \\
\hline$C(9)$ & $22(2)$ & $18(2)$ & $21(2)$ & $2(2)$ & $6(2)$ & $-4(2)$ \\
\hline$C(10)$ & $24(2)$ & $22(2)$ & $28(3)$ & $1(2)$ & $3(2)$ & $3(2)$ \\
\hline$C(11)$ & $32(3)$ & $22(2)$ & $35(3)$ & $-5(2)$ & $10(2)$ & $3(2)$ \\
\hline$C(12)$ & $37(3)$ & $24(2)$ & $30(3)$ & $-10(2)$ & $8(2)$ & $-6(2)$ \\
\hline$C(13)$ & $24(3)$ & $31(3)$ & $22(2)$ & $0(2)$ & $6(2)$ & $-2(2)$ \\
\hline$C(14)$ & $23(2)$ & $19(2)$ & $21(2)$ & $0(2)$ & $5(2)$ & $0(2)$ \\
\hline$C(15)$ & $28(3)$ & $27(3)$ & $26(3)$ & $3(2)$ & $-10(2)$ & $-5(2)$ \\
\hline$C(16)$ & $39(3)$ & $32(3)$ & $56(4)$ & $12(3)$ & $-13(3)$ & $2(3)$ \\
\hline$C(17)$ & $24(3)$ & $31(3)$ & $24(3)$ & $4(2)$ & $-11(2)$ & $-4(2)$ \\
\hline $\mathrm{C}(18)$ & $40(3)$ & $27(3)$ & $26(3)$ & $-1(2)$ & $-18(2)$ & $-5(2)$ \\
\hline$C(19)$ & $34(3)$ & $39(3)$ & $22(3)$ & $-7(2)$ & $-7(2)$ & $2(2)$ \\
\hline$C(20)$ & $37(3)$ & $36(3)$ & $18(2)$ & $5(2)$ & $-7(2)$ & $-9(2)$ \\
\hline$C(27)$ & $27(3)$ & $22(2)$ & $19(2)$ & $-4(2)$ & $1(2)$ & $-5(2)$ \\
\hline$C(28)$ & $29(3)$ & $25(2)$ & $25(3)$ & $-4(2)$ & 7 (2) & $-5(2)$ \\
\hline C (29) & $27(3)$ & $35(3)$ & $28(3)$ & $-4(2)$ & $-6(2)$ & $-3(2)$ \\
\hline
\end{tabular}


Table 10. Hydrogen coordinates ( $\mathrm{x}$ 10^4) and isotropic displacement parameters $\left(A^{\wedge} 2 \times 10^{\wedge} 3\right)$ for 8 .

\begin{tabular}{|c|c|c|c|c|}
\hline & $\mathrm{x}$ & y & z & $\mathrm{U}(\mathrm{eq})$ \\
\hline $\mathrm{H}(01)$ & $3650(50)$ & $1140(30)$ & $9520(40)$ & $62(18)$ \\
\hline $\mathrm{H}(1)$ & $3440(40)$ & $2690(20)$ & $8190(30)$ & 21 \\
\hline $\mathrm{H}(3)$ & 6924 & 2901 & 8525 & 29 \\
\hline $\mathrm{H}(4)$ & 8036 & 3995 & 8682 & 37 \\
\hline H (5) & 6866 & 5075 & 8871 & 39 \\
\hline $\mathrm{H}(6)$ & 4558 & 5051 & 8889 & 37 \\
\hline H ( 7) & 3421 & 3957 & 8747 & 30 \\
\hline $\mathrm{H}(8)$ & $5800(50)$ & $1970(20)$ & $8470(30)$ & 22 \\
\hline $\mathrm{H}(10)$ & 5810 & 601 & 7993 & 30 \\
\hline $\mathrm{H}(11)$ & 5201 & -243 & 6826 & 36 \\
\hline $\mathrm{H}(12)$ & 3547 & 25 & 5681 & 36 \\
\hline $\mathrm{H}(13)$ & 2465 & 1151 & 5743 & 31 \\
\hline H (14) & 3080 & 2003 & 6915 & 25 \\
\hline $\mathrm{H}(16 \mathrm{~A})$ & 6926 & 819 & 10230 & 63 \\
\hline $\mathrm{H}(16 \mathrm{~B})$ & 5732 & 397 & 10745 & 63 \\
\hline $\mathrm{H}(16 \mathrm{C})$ & 6904 & 742 & 11393 & 63 \\
\hline $\mathrm{H}(17)$ & 6815 & 2292 & 10220 & 32 \\
\hline $\mathrm{H}(18)$ & 5130 & 3234 & 10746 & 37 \\
\hline $\mathrm{H}(19)$ & 3305 & 2582 & 11641 & 38 \\
\hline $\mathrm{H}(20)$ & 3835 & 1226 & 11632 & 36 \\
\hline H (27) & 1313 & 1666 & 7985 & 27 \\
\hline $\mathrm{H}(28 \mathrm{~A})$ & 1318 & 309 & 9001 & 40 \\
\hline $\mathrm{H}(28 \mathrm{~B})$ & 2569 & 630 & 8431 & 40 \\
\hline $\mathrm{H}(28 \mathrm{C})$ & 1251 & 407 & 7841 & 40 \\
\hline $\mathrm{H}(29 \mathrm{~A})$ & -778 & 1192 & 7927 & 45 \\
\hline $\mathrm{H}(29 \mathrm{~B})$ & -817 & 1866 & 8667 & 45 \\
\hline $\mathrm{H}(29 \mathrm{C})$ & -788 & 1051 & 9079 & 45 \\
\hline
\end{tabular}

